\title{
6-man chess and zugzwangs
}

Article

Accepted Version

Bleicher, E. and Haworth, G. M. (2010) 6-man chess and zugzwangs. Lecture Notes in Computer Science, 6048. pp. 123-135. ISSN 0302-9743 doi: https://doi.org/10.1007/978-3642-12993-3_12 Available at https://centaur.reading.ac.uk/4518/

It is advisable to refer to the publisher's version if you intend to cite from the work. See Guidance on citing.

To link to this article DOI: http://dx.doi.org/10.1007/978-3-642-12993-3_12

Publisher: Springer-Verlag

Publisher statement: The original publication is available at www.springer.com/Incs

All outputs in CentAUR are protected by Intellectual Property Rights law, including copyright law. Copyright and IPR is retained by the creators or other copyright holders. Terms and conditions for use of this material are defined in the End User Agreement.

\section{$\underline{\text { www.reading.ac.uk/centaur }}$}

\section{CentAUR}

Central Archive at the University of Reading

Reading's research outputs online 


\title{
6-man Chess and Zugzwangs
}

\author{
Eiko Bleicher ${ }^{1}$ and Guy Haworth ${ }^{2}$ \\ ${ }^{1}$ bleicher@k4it.de \\ ${ }^{2}$ School of Systems Engineering, University of Reading, UK \\ guy.haworth@bnc.oxon.org
}

\begin{abstract}
With 6-man Chess essentially solved, the available 6-man Endgame Tables (EGTs) have been scanned for zugzwang positions where, unusually, having the move is a disadvantage. Review statistics together with some highlights and positions are provided here: the complete information is available on the ICGA website. An outcome of the review is the observation that the definition of zugzwang should be revisited, if only because the presence of en passant capture moves gives rise to three new, asymmetric types of zugzwang.
\end{abstract}

Keywords: chess, endgame tables, maximal, Nalimov, zugzwang

\section{Introduction}

Six-man Chess is essentially solved and the Nalimov [1] Endgame Tables (EGTs) to DTM, Depth to Mate, have been widely promulgated for some time [2] ${ }^{1}$. The corpus of perfect information is a challenge to datamine for both helpful guidelines and for the pathological positions - the deep, exceptional, bizarre and amusing.

A zugzwang position is defined here as one where the side to move would prefer that it were the other side's turn to move. They are remarkable in themselves and the 'zug', much sought after by composers, is a running theme in the Study community [3-9]. The zug is also the counterexample to the assumption of the Null Move Heuristic that having the move is an advantage. When 5-man chess was solved, the two sets of respectively DTM and DTC ${ }^{2}$ [10] EGTs were searched [11] for zugs ${ }^{3}$. A later review of Thompson's 6-man pawnless DTC EGTs also included a zug search [12].

The zugzwang search reported here is almost entirely of the highly available Nalimov DTM EGTs [2], [13]. The search was carried out via the web using Bourzutschky's GTBGEN and the first author's EGTs and JAVA code. Unpublished FEG DTM EGTs [14] for 5-1(p) chess and DTC EGTs for 6-man chess [15-16] exist and Bourzutschky has supplied [16] a zug review of 5-1(p) chess from the latter.

Section 2 considers zugzwang definitions and identifies three new types of zug: section 3 covers logistics. Section 4 is a summary of the main findings. Tables 1-5 list various illustrative statistics and positions; the full details are available via the ICGA website [17] which will host the evolving story of the zugzwang.

\footnotetext{
${ }^{1}$ Essentially because positions with castling rights are not yet included in EGTs.

${ }^{2}$ DTC $\equiv$ Depth to Conversion, i.e. to force-change and/or Mate.

${ }^{3}$ Incidentally providing a partial cross-check of agreement between the two sets of EGTs.
} 


\section{Definitions of The Zugzwang}

Zugzwang is defined [18] as pressure to take action and a zugzwang position is defined to be one where this pressure is unwelcome - where the first player would rather 'pass the position across'. ${ }^{4}$ However, in [19] a zugzwang position is defined as 'a position in which whoever has the move would obtain a worse result than if it were the opponent's turn to play'. Note that this now involves the $2^{\text {nd }}$ player's perspective and focuses only on the outcome without considering its achievement or likelihood. Other authorities refer to zugzwangs as reciprocal or mutual zugzwangs. The words whoever, reciprocal and mutual suggest a symmetry, perhaps assuming incorrectly that the $2^{\text {nd }}$ player can always pass back the $1^{\text {st }}$ position to the $1^{\text {st }}$ player.

Consider the en passant zone EPZ of Chess, i.e., those positions where there is an en passant capture option. Let $p 1 \in \mathrm{EPZ}$ : what now are positions $p 2$ and $p 3$ ? The proposal here ${ }^{5}$ is to clarify this situation by formalising the notions of passing over or losing the move as one of playing a null move or nulling. Now the rules of chess define $p 2$ and $p 3$ : the e.p.-capture option in $p 1$ disappears if not played immediately, $p 2$ does not feature any e.p.-capture option as the $1^{\text {st }}$ player has not moved a Pawn two squares, and similarly, $p 3$ is $p 1$ without e.p.-capture option. Positions in EPZ are $0.62 \%$ of those in their endgame and zugs in EPZ comprise $0.22 \%$ of the total.

Let the Level A zugzwang, our focus here, be described in these terms:

a) positions are valued from $1^{\text {st }}$ player's perspective: loss (0), draw (1), win (2),

b) if there is force-symmetry and/or no e.p., $1^{\text {st }}$ player is assumed to be White

Black plays first in 11 Table 1 positions (Zs 04-5, 07-9, 12, 22-4, 26-27),

c) $1^{\text {st }}$ player in position $p 1$ (value $v 1$ ) nulls to $p 2$ (value $v 2$ ) iff $v 2>v 1,{ }^{6}$

d) $2^{\text {nd }}$ player may or may not, c.f. Table 1's Z08-9, null to $p 3$, value $v 3 \leq v 2$,

e) if $p 3 \equiv p 1, v 3 \equiv v 1$. If $p 3 \neq p 1, p 3$ is 'stalemate' or $v 3 \leq v 1$.

Fig. 1 is formatted so that the $1^{\text {st }}$ player nulls 'to the right' and the $2^{\text {nd }}$ player nulls to the left to increase value to themselves. Clearly, in addition to the three familiar zug types A1-3, ${ }^{7}$ we now have, exclusively in the EPZ, just three more types A4-6: ${ }^{8}$

1) type $\mathrm{A} 1 \equiv{ }^{\prime} 121$ ' $\equiv$ 'draw-win-draw', q.v. Z01 in Table 1

2) type $\mathrm{A} 2 \equiv{ }^{\prime} 010$ ' $\equiv$ 'loss-draw-loss', q.v. Z02: A1-3 are no net gain for player 1

3) type $\mathrm{A} 3 \equiv$ ' 020 ' $\equiv$ 'loss-win-loss', the full-point zug, q.v. Z03

4) type A4 $\equiv{ }^{\prime} 120$ ' $\equiv$ 'draw-win-loss', a net loss for player 1, q.v. Z05-6

5) type $\mathrm{A} 5 \equiv{ }^{\prime} 021$ ' $\equiv$ 'loss-win-draw', a net gain for player 1, q.v. Z07

6) type A6 $\equiv{ }^{\prime} 01(1)^{\prime} \equiv$ 'loss-draw(-draw)', a net gain for player $1^{9}$, q.v. Z08-9

Clearly, type A4-6 zugs are asymmetric. Considered only in terms of the first two positions, A4 becomes A1, A5 becomes A3 and A6 becomes A2.

\footnotetext{
${ }^{4}$ Not strictly possible, as in 'passing the position across', the side to move changes.

${ }^{5}$ Our project log notes that Bourzutschky proposed the Null Move concept on 2005-05-31.

${ }^{6}$ Value is calculated as normally, assuming that the option of a null move is not available.

${ }^{7}$ Types are distinguished by the sequence $v_{1}-v_{2}-v_{3}$ rather than just by the sequence $v_{1}-v_{2}$.

${ }^{8}$ Unless position $p 3$ is stalemate, its value $v 3 \leq v 1$ as the $1^{\text {st }}$ player has one less move in $p 3$.

${ }^{9}$ The $2^{\text {nd }}$ player may even prefer to play on rather than stalemate their opponent.
} 


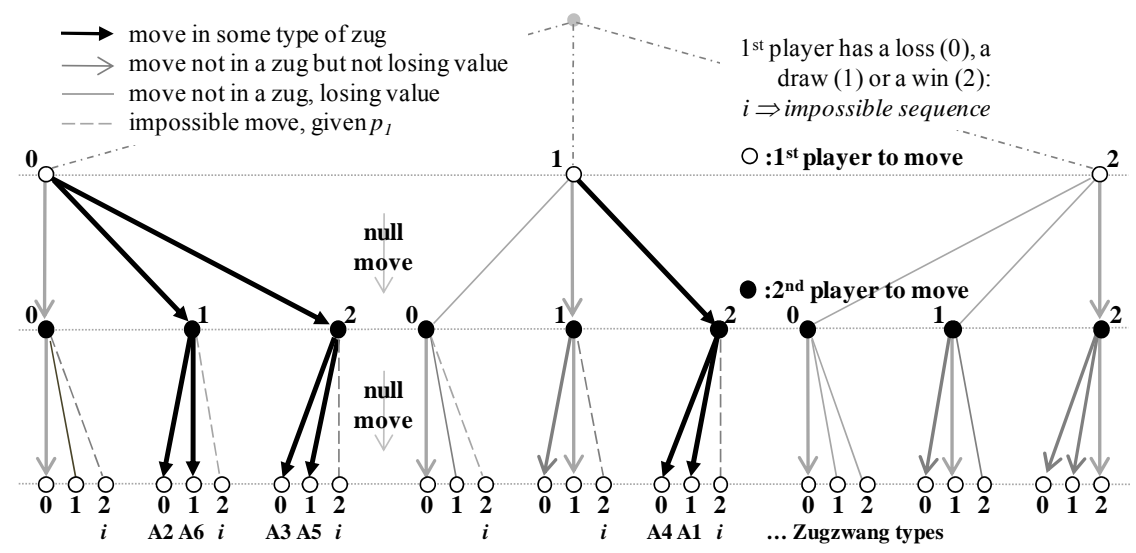

Fig. 1. The six types of Level A zugzwang: the familiar types A1-3 and the 'EPZ types' A4-6.

Table 1. Some examples of zugzwangs of types A1-6. ${ }^{10,11}$

\begin{tabular}{|c|c|c|c|c|c|c|c|c|c|}
\hline \multirow{3}{*}{$\begin{array}{c}\text { id } \\
\mathrm{Z} 01\end{array}$} & \multirow{3}{*}{$\frac{\text { Endgame }}{\mathrm{KPK}}$} & \multirow[b]{2}{*}{ Position } & \multicolumn{2}{|c|}{$\overline{\text { Zug }}$} & \multicolumn{3}{|c|}{ value and DT $x$} & \multirow[b]{2}{*}{$x$} & \multirow[b]{2}{*}{ Flag } \\
\hline & & & & & p1 & p2 & p3 & & \\
\hline & & $1 \mathrm{k} 6 / 1 \mathrm{P} 6 / 2 \mathrm{~K} 5 / 8 / 8 / 8 / 8 / 8 \mathrm{w}$ & $\mathrm{A} 1$ & 121 & $=$ & +2 & $=$ & $\mathrm{Z}$ & \\
\hline Z02 & KBKP & $8 / 8 / 8 / 8 / 8 / 8 / 1 \mathrm{pK} 5 / \mathrm{kB} 6 \mathrm{w}$ & $\mathrm{A} 2$ & 010 & -1 & $==$ & -1 & $\mathrm{Z}$ & $\mathrm{m}$ \\
\hline Z03 & КРКР & $8 / 1 \mathrm{pK} 5 / \mathrm{kP} 6 / 8 / 8 / 8 / 8 / 8 \mathrm{w}$ & A3 & 020 & -1 & +1 & -1 & $\mathrm{Z}$ & $\mathrm{m}$ \\
\hline Z04 & KPPKP & $8 / 8 / 8 / 3 \mathrm{k} 4 / 2 \mathrm{pP} 4 / 2 \mathrm{~K} 5 / 1 \mathrm{P} 6 / 8 \mathrm{~b}-\mathrm{d} 3$ & $\mathrm{~A} 2$ & 010 & -25 & $=$ & -15 & $\mathrm{M}$ & \\
\hline Z05 & КВРКРP & 8/8/8/1p6/1Pp5/8/4K3/2kB4 b - b3 & A4 & 120 & $=$ & +21 & -20 & M & $\mathrm{u}$ \\
\hline Z06 & КРРКРР & 8/1p6/1k6/pP6/K7/P7/8/8 w - a6 & A4 & 120 & $=$ & +21 & -30 & $\mathrm{M}$ & $\mathrm{m}$ \\
\hline Z07 & КР(5)КР(4) & 8/8/8/2p5/1pP1p3/kP2P3/Pp1P4/1K6 b - c3 & A5 & 021 & -0 & +1 & $==$ & $\mathrm{Z}$ & \\
\hline Z08 & KRPKPP & 8/8/8/8/pP6/p7/k1K5/1R6 b - b3 & A6 & 011 & -0 & $=$ & $==$ & $\mathrm{Z}$ & $\mathrm{u}$ \\
\hline Z09 & КРРРКР & 8/8/8/8/1pP5/kP6/P7/K7 b - c3 & A6 & 011 & -0 & $==$ & $==$ & $\mathrm{Z}$ & $\mathrm{u}$ \\
\hline Z10 & KRNKNN & 8/8/8/8/2n5/1n6/R1N5/3K1k2 w & $\mathrm{A} 2$ & 010 & -1 & $=$ & -1 & $\mathrm{M}$ & \\
\hline Z11 & KRBNKNN & 8/8/8/8/2n5/1n6/R1N5/1B1K1k2 u & A3 & 020 & -1 & $+38(+)$ & -1 & $\mathrm{M}$ & \\
\hline Z12 & KBPKNP & $2 \mathrm{n} 5 / 8 / 8 / 2 \mathrm{~B} 5 / 1 \mathrm{Pp} 5 / \mathrm{k} 1 \mathrm{~K} 5 / 8 / 8 \mathrm{~b}-\mathrm{b} 3$ & $\mathrm{~A} 2$ & 010 & -18 & $=$ & -18 & $\mathrm{M}$ & $\mathrm{i}$ \\
\hline Z13 & KQBKNP & $8 / 1 \mathrm{~K} 6 / 8 / 2 \mathrm{k} 5 / \mathrm{Q} 1 \mathrm{Bn} 4 / 8 / 2 \mathrm{p} 5 / 8 \mathrm{w}$ & Al & 121 & $=$ & +21 & $=$ & $\mathrm{M}$ & $\mathrm{U}$ \\
\hline Z14 & KQNKBN & 8/8/8/8/8/nkb5/1N6/Q1K5 w & A1 & 121 & $=$ & +35 & $=$ & $\mathrm{M}$ & $\mathrm{U}$ \\
\hline Z15 & KQNKNN & $5 / 8 / 2 \mathrm{kN} 4 / \mathrm{K} 7 \mathrm{w}$ & $\mathrm{A} 1$ & 121 & $=$ & +35 & $=$ & $\mathrm{M}$ & $\mathrm{U}$ \\
\hline Z16 & KQRKBB & $8 / 8 / 6 \mathrm{~b} 1 / 2 \mathrm{R} 5 / 3 \mathrm{~K} 4 / 4 \mathrm{Q} 3 / 5 \mathrm{~b} 2 / 5 \mathrm{k} 2 \mathrm{w}$ & Al & 121 & $=$ & +26 & $=$ & $\mathrm{M}$ & $\mathrm{U}$ \\
\hline Z17 & KQRKRB & 2r5/R1Q5/b7/8/8/2K5/8/1k6 w & Al & 121 & $=$ & +17 & $=$ & $\mathrm{M}$ & $\mathrm{U}$ \\
\hline Z18 & KQBPKB & $1 \mathrm{k} 6 / 1 \mathrm{P} 6 / \mathrm{K} 7 / 1 \mathrm{Q} 6 / \mathrm{B} 1 \mathrm{~b} 5 / 8 / 8 / 8 \mathrm{w}$ & Al & 121 & $=$ & +9 & $=$ & $\mathrm{M}$ & $\mathrm{U}$ \\
\hline Z19 & KQBPKP & 8/8/8/8/8/1p6/1PQ5/kBK5 w & Al & 121 & $=$ & +11 & $=$ & $\mathrm{M}$ & $\mathrm{U}$ \\
\hline Z20 & KRRRKQ & 8/8/8/8/8/4q1k1/2R5/1R1K3R w & Al & 121 & $=$ & +22 & $=$ & $\mathrm{M}$ & $\mathrm{U}$ \\
\hline Z21 & KBPKNP & $3 \mathrm{n} 4 / 8 / 8 / 5 \mathrm{pP} 1 / 8 / 8 / 8 / 1 \mathrm{kBK} 4 \mathrm{w}-\mathrm{f} 6$ & Al & 121 & $=$ & 32 & $=$ & $\mathrm{M}$ & \\
\hline Z22 & KBPKNP & 8/8/3B2n1/K7/1pP5/k7/8/8b-c3 & $\mathrm{A} 2$ & 010 & -32 & $=$ & -32 & $\mathrm{M}$ & \\
\hline Z23 & KNPKPP & 8/8/8/p7/Pp6/3N4/3K4/k7 b - a3 & $\mathrm{A} 2$ & 010 & -25 & $=$ & -25 & $\mathrm{M}$ & \\
\hline Z24 & KRPKPP & $8 / 1 \mathrm{~K} 6 / 1 \mathrm{p} 6 / 2 \mathrm{k} 5 / 1 \mathrm{pP} 5 / 8 / 8 / 2 \mathrm{R} 5 \mathrm{~b}-\mathrm{c} 3$ & A2 & 010 & -21 & $=$ & -21 & $\mathrm{M}$ & \\
\hline Z25 & КВPPКP & 1BK5/8/k7/Pp6/8/P7/8/8 w - b6 & Al & 121 & $=$ & +17 & $=$ & $\mathrm{M}$ & \\
\hline Z26 & KNPPKP & 8/8/K7/PNk5/Pp6/8/8/8 b - a3 & A2 & 010 & -23 & $=$ & -23 & $\mathrm{M}$ & \\
\hline Z27 & КPРPKP & 8/8/1k6/1P6/KPp5/8/P7/8 b - b3 & A2 & 010 & -17 & $=$ & -17 & $\mathrm{M}$ & \\
\hline
\end{tabular}

${ }^{10}$ Values from $1^{\text {st }}$ player's perspective: + win, $=$ draw, == stalemate, - loss.

${ }^{11}$ Flags: $m$ maxDT $x, i$ inaccessible, $s$ symmetric, $u$ unique of type, $U$ unique in endgame. 


\section{Enumeration: Endgames and Zugzwang Occurrences}

These notes explain the lexical ordering of men and of endgames, and the principles used for counting the occurrences of zugzwangs.

The men are listed in the strength order K-Q-R-B-N-P. White has at least as many men as Black. In $m-m$ endgames, White's lead men are at least as strong as Black's.

No attempt is made to eliminate unreachable positions in EGT statistics: this is usual as there is no general algorithm. With this limitation, the count is of FENdistinct and functionally unique zugzwangs. Thus, no zugzwang can be physically transformed into any other. The following subtleties should be noted:

a) For force-symmetric zugs $z \notin \mathrm{EPZ}$, type A1 and A2 zugs are equivalent: the count of A2 zugs is shown in brackets,

b) Given force symmetry, A3 zugs usually appears in two physical forms: - the two physical versions were identified ${ }^{12}$ and counted as one here,

c) When both Kings are on a1-h8 or a8-h1 in pawnless endgames: Nalimov has both physical versions of the position if there are two; the two physical versions were identified and counted as one here,

d) When e.p.-capture has been enabled but is actually illegal, q.v. Z12: the position is counted here as different from that without the e.p., $1^{\text {st }}$ player would have to realize that the e.p.-option is illusory, FIDE's recently reworded Article 9.2 now seeks to ignore the e.p. [20-22],

e) An example of an unreachable zug: position Z12 also implies the prior 1. b2-b4, impossible on two grounds: before 1. b2-b4, the side not to move, Black, is in (double) check, check from a Pawn on its home square is itself impossible, therefore, the position prior to $\mathrm{Z} 12$ is also unreachable.

We note one small, historical and now resolved hiatus with respect to these results. The identification of zugs of types A4-6 was a serendipitous accident ${ }^{13}$ and was initially regarded as a bug by GTBGEN's author Marc Bourzutschky.

When the 'last 16' 3-3p endgames to KPPKPP were published, both by MB converting his FEG EGTs to Nalimov's format ${ }^{14}$ [23] and by Nalimov returning a disc to Hernandez [24], MB discovered that although he had anticipated a 2-byte format for the KQPK(B/R)P EGTs in GTBGEN, Nalimov had in fact discovered that the 1-byte format would suffice [25]. MB realigned with Nalimov and removed the ability to detect type A4-6 zugs before a GTBGEN was provided that could address these two EGTs. MB now advises [16] that no type A4-6 KQPKBP or KQPKRP zug exists.

Results for Level A zugs have been published as a set of files [17]. Table 2 provides summary statistics for the six blocks of 6-man zugs, 3-3(p), 4-2(p) and 5-1(p). There are $2935-1 p$ zugs, 261 created by a touring Knight being on the wrong foot.

Table 3 details the occurrences of e.p. zugs including the new types of zug. Table 4 lists in lexical order all endgames with A3 zugs, together with an example of their deepest such zug. Table 5 is a miscellany of exemplar positions: an A2 e.p. zug with

12 The identification of symmetries and equivalences was done by the first author's code.

${ }^{13}$ The serendipitous accident has its place of honour in the history of discovery.

${ }^{14}$ After reverse-engineering the unpublished format of the FEG EGT data format. 
p3's depth less than p1's (P03), an A3 e.p. zug (N01), A2 and A3 zugs dezugged by the addition of an e.p. opportunity (N02-3) or castling option (P01), zugs unaffected (P02) or created (N04-8) by giving castling rights to the $1^{\text {st }}$ or $2^{\text {nd }}$ player, 5-1p zugs (P06-13), 7-man zugs (B01-6) and Zugzwang Studies (S01-12) featuring zugs. Elkies [26] composed N01-10 and Bourzutschky [15-16] found B01-6.

Table 2. Level A zugs: summary statistics for each 6-man endgame group. ${ }^{15,16}$

\begin{tabular}{|c|c|c|c|c|c|c|c|c|}
\hline & Item \Group & $3-3$ & $4-2$ & $5-1$ & 3-3p & 4-2p & 5-1p & Total \\
\hline 01 & Endgames & 55 & 80 & 35 & 65 & 95 & 35 & 365 \\
\hline 02 & No zugs & 12 & 50 & 35 & 12 & 29 & 28 & 166 \\
\hline 03 & No A1 zugs & 12 & 50 & 35 & 12 & 30 & 28 & 167 \\
\hline 04 & No A2 zugs & $31(+9)$ & 71 & - & $22(+3)$ & 51 & - & $175(+12)$ \\
\hline 05 & No A3 zugs & 55 & 80 & - & 50 & 67 & - & 252 \\
\hline 06 & A unique zug & 4 & 1 & 0 & 1 & 2 & 0 & 8 \\
\hline 07 & One A1 zug & 4 & 1 & 0 & 1 & 4 & 0 & 10 \\
\hline 08 & One A2 zug & 1 & 2 & - & 1 & 6 & - & 10 \\
\hline 09 & One A3 zug & 0 & 0 & - & 6 & 3 & - & 9 \\
\hline 10 & A1-A6 zugs & 27,597 & 20,017 & 0 & 380,363 & 478,682 & 293 & 906,952 \\
\hline 11 & A1 zugs & 27,470 & 8,434 & 0 & 361,725 & 373,479 & 293 & 771,401 \\
\hline 12 & A2 zugs & 127 & 11,583 & - & 15,543 & 105,069 & - & 132,322 \\
\hline 13 & A 3 zugs & 0 & 0 & - & 2,700 & 133 & - & 2,833 \\
\hline 14 & A4 zugs & - & - & - & 394 & 0 & - & 394 \\
\hline 15 & A5 zugs & - & - & - & 0 & 0 & - & 0 \\
\hline 16 & A6 zugs & - & - & - & 1 & 1 & 一 & 2 \\
\hline
\end{tabular}

Table 3. Statistics for the fourteen endgames with e.p.-zugs.

\begin{tabular}{|c|c|c|c|c|c|c|c|c|c|c|c|c|c|c|}
\hline Endgame & \multicolumn{2}{|c|}{ e.p.-zugs } & \multicolumn{2}{|c|}{ A1 } & A2 & $\begin{array}{l}2 \\
\text { btm }\end{array}$ & A3 & $\begin{array}{l}3 \\
\text { btm }\end{array}$ & $\begin{array}{r}\text { A } \\
\text { wtm }\end{array}$ & A4 & & $\begin{array}{l}\text { A5 } \\
\text { a btm }\end{array}$ & \multicolumn{2}{|c|}{ A6 } \\
\hline КРPКР & 35 & 20 & 35 & 0 & 0 & 20 & 0 & 0 & 0 & 0 & 0 & 0 & 0 & 0 \\
\hline КВРКВР & 10 & -- & 10 & -- & 0 & -- & 0 & -- & 0 & -- & 0 & -- & 0 & - - \\
\hline KBPKNP & 130 & 5 & 130 & 2 & 0 & 3 & 0 & 0 & 0 & 0 & 0 & 0 & 0 & 0 \\
\hline КВРКРР & 18 & 18 & 18 & 4 & 0 & 13 & 0 & 0 & 0 & 1 & 0 & 0 & 0 & 0 \\
\hline KNPKNP & 156 & -- & 156 & -- & 0 & -- & 0 & -- & 0 & -- & 0 & - - & 0 & -- \\
\hline KNPKPP & 250 & 19 & 250 & 17 & 0 & 2 & 0 & 0 & 0 & 0 & 0 & 0 & 0 & 0 \\
\hline КРРКРР & 1,301 & -- & 869 & -- & 39 & -- & 0 & -- & 393 & -- & 0 & -- & 0 & -- \\
\hline KQPKQP & 75 & -- & 72 & -- & 3 & -- & 0 & -- & 0 & -- & 0 & -- & 0 & -- \\
\hline KRPKBP & 20 & 7 & 20 & 0 & 0 & 7 & 0 & 0 & 0 & 0 & 0 & 0 & 0 & 0 \\
\hline KRPKNP & 27 & 14 & 27 & 0 & 0 & 14 & 0 & 0 & 0 & 0 & 0 & 0 & 0 & 0 \\
\hline KRPKPP & 0 & 2 & 0 & 0 & 0 & 1 & 0 & 0 & 0 & 0 & 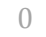 & 0 & 0 & 1 \\
\hline КВРРКР & 1 & 0 & 1 & 0 & 0 & 0 & 0 & 0 & 0 & 0 & $\Omega$ & 0 & 0 & 0 \\
\hline KNPPKP & 0 & 1 & 0 & 0 & 0 & 1 & 0 & 0 & 0 & 0 & 0 & 0 & 0 & 0 \\
\hline КРРРКР & 8 & 2 & 8 & 0 & 0 & 1 & 0 & 0 & 0 & 0 & 0 & 0 & 0 & 1 \\
\hline Totals & 2,031 & 88 & 1,596 & 23 & 42 & 62 & 0 & 0 & 393 & 1 & 0 & 0 & 0 & 2 \\
\hline
\end{tabular}

${ }^{15}(+n)$ indicates that there are $n$ endgames whose A2 zugs merely mirror their A1 zugs.

${ }^{16}$ The eight zugzwangs which are unique across their endgames are Z12-Z19 in Table 1. 
Table 4. The 43 endgames with full-point type A3 zugs: maxDTM examples. ${ }^{17,18,19}$

\begin{tabular}{|c|c|c|c|c|c|c|c|}
\hline \multirow[b]{2}{*}{ id } & \multirow[b]{2}{*}{ Endgame } & \multirow[b]{2}{*}{ Position } & \multicolumn{3}{|c|}{ val / DTM } & \multicolumn{2}{|l|}{ Total } \\
\hline & & & p1 & p2 & p3 & Depth & Flag \\
\hline F01 & KBPKNP & $\mathrm{k} 7 / \mathrm{Bp} 4 \mathrm{n} 1 / 1 \mathrm{~K} 1 \mathrm{P} 4 / 8 / 8 / 8 / 8 / 8 \mathrm{w}$ & -25 & +16 & -25 & 41 & \\
\hline F02 & КВРКРР & $8 / 8 / 8 / 8 / 3 \mathrm{~K} 1 \mathrm{k} 1 \mathrm{p} / 3 \mathrm{P} 2 \mathrm{p} 1 / 6 \mathrm{~B} 1 / 8 \mathrm{w}$ & -57 & +28 & -57 & 85 & \\
\hline F03 & KNPKNP & 8/8/8/8/8/K1k5/P1p5/n1N5 w & -13 & +24 & -13 & 37 & $\mathrm{u}$ \\
\hline F04 & KNPKPP & $8 / 8 / 8 / 8 / 4 \mathrm{kp} 1 \mathrm{p} / 3 \mathrm{~N} 4 / 2 \mathrm{KP} 4 / 8 \mathrm{w}$ & -71 & +26 & -71 & 97 & \\
\hline F05 & КРРКРР & 8/8/8/8/p4p2/k1P5/2K1P3/8 w & -17 & +103 & -17 & 120 & c.f. '18' \\
\hline F06 & KQNKQP & $8 / 8 / 8 / 3 N 4 / 8 / k 2 p 4 / 3 q 4 / K Q 6 w$ & -14 & +6 & -14 & 20 & $\mathrm{u}$ \\
\hline F07 & KQNKRP & QN6/Kpk5/1r6/8/8/8/8/8 w & -8 & +32 & -8 & 40 & $\mathrm{u}$ \\
\hline F08 & KQPKQP & $8 / 8 / 8 / 1 \mathrm{Pq} 5 / 8 / 1 \mathrm{~K} 1 \mathrm{Q} 4 / 5 \mathrm{p} 2 / 2 \mathrm{k} 5 \mathrm{w}$ & -24 & +95 & -24 & 119 & \\
\hline F09 & KQPKRB & $2 \mathrm{~K} 1 \mathrm{k} 3 / 2 \mathrm{P} 5 / 8 / 8 / 8 / 8 / 1 \mathrm{r} 2 \mathrm{~b} 3 / 4 \mathrm{Q} 3 \mathrm{w}$ & -20 & +15 & -20 & 35 & $\mathrm{u}$ \\
\hline F10 & KQPKRP & 8/8/8/1Q6/8/1pP5/2k2r2/K7 w & -12 & +42 & -12 & 54 & \\
\hline F11 & KRNKNP & 8/8/8/8/8/p7/N2k4/RK1n4 w & -1 & +28 & -1 & 29 & $\mathrm{u}$ \\
\hline F12 & KRNKPP & $8 / 8 / 8 / 2 \mathrm{~N} 5 / 8 / 7 \mathrm{p} / \mathrm{k} 5 \mathrm{pR} / 2 \mathrm{~K} 5 \mathrm{w}$ & -33 & +17 & -33 & 50 & \\
\hline F13 & KRPKBP & 8/8/8/8/8/2p5/2Pk4/1KR1b3 w & -15 & +30 & -15 & 45 & \\
\hline F14 & KRPKNP & 8/8/8/8/3n4/k7/p1P5/K1R5 w & -1 & +33 & -1 & 34 & $\mathrm{u}$ \\
\hline F15 & KRPKPP & 8/8/6R1/k1P5/2K5/7p/6p1/8 w & -71 & +9 & -71 & 80 & \\
\hline F16 & KBBPKQ & 8/8/8/8/1K6/BBP5/8/qk6 w & -102 & +13 & -102 & 115 & \\
\hline F17 & KBNPKB & $\mathrm{K} 7 / \mathrm{P} 1 \mathrm{k} 5 / 8 / 8 / 8 / 8 / 6 \mathrm{~N} 1 / 5 \mathrm{~B} 1 \mathrm{~b} w$ & -2 & +14 & -2 & 16 & \\
\hline F18 & KBNPKN & $\mathrm{BK} 1 \mathrm{n} 4 / \mathrm{NP} 1 \mathrm{k} 4 / 8 / 8 / 8 / 8 / 8 / 8 \mathrm{w}$ & -1 & +32 & -1 & 33 & \\
\hline F19 & KBPPKB & $\mathrm{K} 7 / \mathrm{P} 1 \mathrm{k} 5 / 8 / 8 / 8 / 8 / 6 \mathrm{P} 1 / 5 \mathrm{~B} 1 \mathrm{~b} \mathrm{w}$ & -2 & +14 & -2 & 16 & \\
\hline F20 & КВРPКР & 8/8/8/8/3k4/1K1p4/1P3P2/B7 w & -18 & +16 & -18 & 34 & \\
\hline F21 & KBPPKQ & $3 \mathrm{~K} 1 \mathrm{kq} 1 / 8 / 4 \mathrm{~PB} 2 / 3 \mathrm{P} 4 / 8 / 8 / 8 / 8 \mathrm{w}$ & -34 & +40 & -34 & 74 & c.f. '19' \\
\hline $\mathrm{F} 22$ & KBPPKR & 8/8/8/8/8/2k5/P1P5/rBK5 w & -18 & +22 & -18 & 40 & \\
\hline F23 & KNNPKN & $\mathrm{K} 1 \mathrm{k} 5 / \mathrm{P} 2 \mathrm{~N} 4 / 4 \mathrm{~N} 3 / 3 \mathrm{n} 4 / 8 / 8 / 8 / 8 \mathrm{w}$ & -1 & +19 & -1 & 20 & \\
\hline F24 & KNPPKN & $\mathrm{K} 7 / \mathrm{P} 1 \mathrm{kN} 4 / 8 / 3 \mathrm{P} 4 / \mathrm{n} 7 / 8 / 8 / 8 \mathrm{w}$ & -2 & +20 & -2 & 22 & \\
\hline F25 & KNPPKP & 8/8/8/K7/P1k5/1p6/3P4/4N3 w & -20 & +23 & -20 & 43 & \\
\hline F26 & KNPPKQ & $1 \mathrm{~K} 1 \mathrm{k} 2 \mathrm{q} 1 / 8 / 2 \mathrm{P} 5 / 3 \mathrm{~N} 4 / 8 / 2 \mathrm{P} 5 / 8 / 8 \mathrm{w}$ & -20 & +45 & -20 & 65 & \\
\hline F27 & KNPPKR & $\mathrm{N} 1 \mathrm{k} 5 / 2 \mathrm{P} 5 / \mathrm{rPK} 5 / 8 / 8 / 8 / 8 / 8 \mathrm{w}$ & -22 & +12 & -22 & 34 & \\
\hline F28 & KPPPKN & $\mathrm{n} 7 / \mathrm{P} 1 \mathrm{k} 5 / \mathrm{K} 7 / \mathrm{PP} 6 / 8 / 8 / 8 / 8 \mathrm{w}$ & -2 & +13 & -2 & 15 & \\
\hline F29 & КРРРКР & 8/8/8/5k2/3K1p2/3P $3 \mathrm{P} / 3 \mathrm{P} 4 / 8 \mathrm{w}$ & -20 & +19 & -20 & 39 & \\
\hline F30 & KPPPKQ & $\mathrm{k} 7 / \mathrm{q} 1 \mathrm{PK} 4 / \mathrm{P} 7 / 8 / 8 / 2 \mathrm{P} 5 / 8 / 8 \mathrm{w}$ & -15 & +19 & -15 & 34 & \\
\hline F31 & KPPPKR & 1K6/1P1k4/1r6/1P6/2P5/8/8/8 w & -21 & +36 & -21 & 57 & \\
\hline F32 & KQNPKN & QN6/KP6/8/nk6/8/8/8/8 w & -1 & +8 & -1 & 9 & \\
\hline F33 & KQNPKQ & $8 / 8 / 8 / 5 \mathrm{~N} 2 / 1 \mathrm{q} 6 / 8 / \mathrm{Q} 2 \mathrm{P} 4 / \mathrm{K} 1 \mathrm{k} 5 \mathrm{w}$ & -3 & +35 & -3 & 38 & \\
\hline F34 & KQPPKQ & 8/8/8/1q6/5P2/P7/Q7/K1k5 w & -4 & +33 & -4 & 37 & \\
\hline F35 & KRBPKB & $\mathrm{K} 7 / \mathrm{P} 1 \mathrm{k} 5 / 8 / 8 / 8 / 8 / 6 \mathrm{R} 1 / 5 \mathrm{~B} 1 \mathrm{~b} \mathrm{w}$ & -2 & +14 & -2 & 16 & \\
\hline F36 & KRBPKN & RK6/B3n3/1Pk5/8/8/8/8/8 w & -2 & +14 & -2 & 16 & $\mathrm{u}$ \\
\hline F37 & KRBPKP & 8/8/8/8/8/1k6/pP6/BRK5 w & -11 & +21 & -11 & 32 & \\
\hline F38 & KRBPKQ & $1 \mathrm{qk} 5 / 8 / \mathrm{RBP} 5 / 8 / 8 / 8 / 8 / 1 \mathrm{~K} 6 \mathrm{w}$ & -41 & +13 & -41 & 54 & $\mathrm{u}$ \\
\hline F39 & KRNPKN & 8/8/8/8/n7/P7/K1k5/RN6 w & -1 & +24 & -1 & 25 & \\
\hline F40 & KRNPKQ & 1K3N2/3R1P2/1kq5/8/8/8/8/8 w & -11 & +16 & -11 & 27 & \\
\hline F41 & KRPPKN & $\mathrm{K} 7 / \mathrm{P} 1 \mathrm{k} 5 / \mathrm{R} 1 \mathrm{P} 5 / 8 / 2 \mathrm{n} 5 / 8 / 8 / 8 \mathrm{w}$ & -1 & +20 & -1 & 21 & \\
\hline F42 & KRPPKQ & 3R4/q7/k1P5/P7/K7/8/8/8 w & -36 & +12 & -36 & 48 & $\mathrm{u}$ \\
\hline F43 & KRPPKR & 8/8/8/8/8/rPK5/1RP5/2k5 w & -26 & +29 & -26 & 55 & \\
\hline
\end{tabular}

${ }^{17}$ The depth of a type A3 or A5 zug is defined as the sum of the depths of $p 1$ and $p 2$.

${ }^{18} \mathrm{KPPKPP}$ zug F05 is the deepest A3 zug with $d t m=120$.

${ }^{19}$ KBPPKQ zug F21 has the maximal DTM-depth 'shallower side' loss (here) with $\mathrm{dtm}=34$. 
Table 5. More didactic positions including 5-1p zugs and e.p. and/or castling effects. ${ }^{20}$

\begin{tabular}{|c|c|c|c|c|c|c|c|c|}
\hline \multirow[b]{2}{*}{ id } & \multirow[b]{2}{*}{ Endgame } & \multirow[b]{2}{*}{ Position } & \multicolumn{3}{|c|}{ DTx } & \multirow[b]{2}{*}{ p3 $x$} & \multirow{2}{*}{\multicolumn{2}{|c|}{ Flag }} \\
\hline & & & Type & p1 & p2 & & & \\
\hline $\mathrm{P} 01$ & KQNKRR & $1 \mathrm{KQNk} 2 \mathrm{r} / 7 \mathrm{r} / 8 / 8 / 8 / 8 / 8 / 8 \mathrm{w}--$ & A1 & $=$ & +54 & $=Z$ & $\mathrm{Z}$ & $\mathrm{c}$ \\
\hline P02 & KRRKRB & $\mathrm{r} 3 \mathrm{~kb} 2 / 1 \mathrm{RK} 4 \mathrm{R} / 8 / 8 / 8 / 8 / 8 / 8 \mathrm{w}--$ & A1 & $=$ & +7 & $=Z$ & $\mathrm{Z}$ & $\mathrm{c}$ \\
\hline P03 & КРPКPP & 8/6p1/4k1P1/4Pp2/3K4/8/8/8 w - f6 & $\mathrm{A} 2$ & -24 & $=$ & 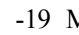 & M & $\mathrm{e}$ \\
\hline P04 & KNNKP & $7 \mathrm{k} / 8 / 5 \mathrm{NK} 1 / 7 \mathrm{p} / 8 / 8 / \mathrm{N} 7 / 8 \mathrm{~b}--$ & B2 & -0 & 1 & $-0 \quad Z$ & $\mathrm{Z} \mathrm{E}$ & B2 \\
\hline P05 & КРРКРР & 8/8/p2k4/6p1/3K2P 1/P7/8/8 b - - & $\mathrm{C}$ & $=$ & $=$ & $=2$ & $\mathrm{Z}$ & $\mathrm{C}$ \\
\hline P06 & КВРPРК & $5 \mathrm{kBK} / 5 \mathrm{P} 1 \mathrm{P} / 7 \mathrm{P} / 8 / 8 / 8 / 8 / 8 \mathrm{w}--$ & A1 & $=$ & +2 & $=z$ & $\mathrm{Z}$ & 5 \\
\hline P07 & КВРРРК & $7 \mathrm{~K} / 5 \mathrm{kBP} / 5 \mathrm{P} 1 \mathrm{P} / 8 / 8 / 8 / 8 / 8 \mathrm{w}--$ & A1 & $=$ & +2 & $=2$ & $\mathrm{Z}$ & 5 \\
\hline P08 & КВРPPK & 8/B1k5/K7/P7/P7/P7/8/8 w - - & $\mathrm{A} 1$ & $=$ & +3 & $=Z$ & Z & 5 \\
\hline P09 & КВPPPK & $1 \mathrm{k} 6 / 8 / \mathrm{KP} 6 / \mathrm{BP} 6 / 1 \mathrm{P} 6 / 8 / 8 / 8 \mathrm{w}--$ & A1 & $=$ & +1 & $=Z$ & $\mathrm{Z}$ & 5 \\
\hline P10 & KNPPPK & $7 \mathrm{~K} / 5 \mathrm{k} 1 \mathrm{P} / 4 \mathrm{~N} 2 \mathrm{P} / 7 \mathrm{P} / 8 / 8 / 8 / 8 \mathrm{w}--$ & A1 & $=$ & +2 & $=2$ & $\mathrm{Z}$ & 5 \\
\hline P11 & КРРPРК & $5 \mathrm{k} 2 / 5 \mathrm{P} 2 / 4 \mathrm{~K} 3 / 7 \mathrm{P} / 7 \mathrm{P} / 7 \mathrm{P} / 8 / 8 \mathrm{w}-$. & A1 & $=$ & +2 & $=z$ & $\mathrm{Z}$ & 5 \\
\hline P12 & KQNPPK & $4 \mathrm{k} 1 \mathrm{KQ} / 5 \mathrm{NPP} / 8 / 8 / 8 / 8 / 8 / 8 \mathrm{w}--$ & A1 & $=$ & +2 & $=2$ & $\mathrm{Z}$ & 5 \\
\hline P13 & KRPPPK & 1k6/1P6/K7/RP6/P7/8/8/8 w - - & A1 & $=$ & +2 & $=Z$ & $\mathrm{Z}$ & 5 \\
\hline N01 & КРРРКРРP & 8/1p6/8/pP4pK/5kP1/P7/8/8 w - a6 & A3 & -1 & +1 & $-1 \quad z$ & $\mathrm{Z}$ & $\mathrm{e}$ \\
\hline N02 & KPPKP & $3 \mathrm{~K} 4 / 8 / 3 \mathrm{k} 4 / 8 / 3 \mathrm{Pp} 3 / 4 \mathrm{P} 3 / 8 / 8 \mathrm{~b}--$ & A2 & -4 & $=$ & $-4 \quad z$ & $\mathrm{Z}$ & $\mathrm{e}$ \\
\hline N03 & КРРКРP & 8/8/8/5pK1/4kPpl/8/7P/8 b - - & $\mathrm{A} 3$ & -1 & +1 & 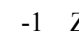 & $\mathrm{Z}$ & $\mathrm{e}$ \\
\hline N04 & KQP(6)KRRBP(3) & Q1K $1 k 2 r / P P P 1 p 2 p / b 1 r 1 P 2 P / 2 p 5 / 2 P 5 / 8 / 8 / 8$ w k - & $-\mathrm{A} 2$ & -1 & $=$ & 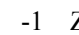 & $\mathrm{Z}$ & $\mathrm{c}$ \\
\hline N05 & KQP(6)KRRBP(5) & Q1K1k2r/PPP 1p2p/bprpP2P/2p5/2P 5/8/8/8 w k - & $-\mathrm{A} 3$ & -1 & +2 & $-1 \quad z$ & $\mathrm{Z}$ & $\mathrm{c}$ \\
\hline N06 & KQP(8)KRRBP(7) & $\mathrm{Q} 1 \mathrm{~K} 1 \mathrm{k} 2 \mathrm{r} / \mathrm{PPP} 1 \mathrm{p} 2 \mathrm{p} / \mathrm{bprpP} 1 \mathrm{pP} / 2 \mathrm{p} 5 / 2 \mathrm{P} 2 \mathrm{pP} 1 / 8 / 5 \mathrm{P} 2 / 8 \mathrm{~b} \mathrm{k} 3$ & A3 & -2 & +2 & $-2 z$ & $\mathrm{Zc}$ & $\mathrm{c} \mathrm{e}$ \\
\hline N07 & KRBNP(3)KRBP & $\mathrm{r} 3 \mathrm{k} 1 \mathrm{KR} / 3 \mathrm{p} 2 \mathrm{~PB} / 3 \mathrm{P} 2 \mathrm{~N} 1 / 3 \mathrm{P} 3 \mathrm{~b} / 8 / 8 / 8 / 8 \mathrm{w} \mathrm{q}-$ & $\mathrm{A} 3$ & -1 & ? & $-1 z$ & $\mathrm{Z}$ & $\mathrm{c}$ \\
\hline N08 K & KRBNP(4)KRBP(3) & ) $\mathrm{r} 3 \mathrm{k} 1 \mathrm{KR} / 3 \mathrm{p} 2 \mathrm{~PB} / 2 \mathrm{pP} 2 \mathrm{~N} 1 / 7 \mathrm{~b} / 1 \mathrm{pP} 5 / 8 / 1 \mathrm{P} 6 / 8 \mathrm{~b} \mathrm{q} \mathrm{c} 3$ & A3 & $-?$ & +1 & $-? \quad Z$ & $\mathrm{Zc}$ & $\mathrm{ce}$ \\
\hline N09 & KPPKP & 8/8/8/3k $2 \mathrm{P} 1 / 4 \mathrm{pKP} 1 / 8 / 8 / 8 \mathrm{w}--$ & B1 & +84 & +25 & $+84 \mathrm{~N}$ & $\mathrm{M} \mathrm{E}$ & B1 \\
\hline N10 & КРРКР & 8/8/3k4/1K1p4/1P6/1P6/8/8 b - - & $\mathrm{C}$ & $=$ & $=$ & $=Z$ & $\mathrm{Z}$ & $\mathrm{C}$ \\
\hline B01 & KNNNKNN & $7 \mathrm{k} / 8 / 4 \mathrm{~N} 3 / 4 \mathrm{NN} 2 / \mathrm{n} 2 \mathrm{~K} 4 / 8 / 8 / 3 \mathrm{n} 4 \mathrm{w}--$ & $\mathrm{A} 1$ & $=$ & +17 & $=Z$ & $\mathrm{Z}$ & $\mathrm{s}$ \\
\hline B02 & KRBBKQB & 8/8/8/8/2b2q2/B7/1R3B2/2k1K3 w - - & $\mathrm{A} 3$ & -96 & +2 & $-96 z$ & $\mathrm{Z}$ & $\mathrm{u}$ \\
\hline B03 & KRBBKQN & 8/5B1q/6R1/3n4/8/8/2KB4/k7 w - - & $\mathrm{A} 3$ & -6 & +2 & $-6 z$ & $\mathrm{Z}$ & \\
\hline B04 & KRRRKRR & 8/8/8/8/8/3Rr3/kr6/2KRR3 w - - & A1 & $=$ & +2 & $=2$ & $\mathrm{Z}$ & $\mathrm{U}$ \\
\hline B05 & KBBBBKQ & $6 \mathrm{~B} 1 / 1 \mathrm{~B} 4 \mathrm{qB} / 5 \mathrm{k} 2 / 8 / 3 \mathrm{~K} 4 / 8 / 6 \mathrm{~B} 1 / 8 \mathrm{w}--$ & $\mathrm{A} 2$ & -35 & $=$ & $-35 z$ & $\mathrm{Z}$ & $\mathrm{s}$ \\
\hline B06 & KBBNNKQ & 8/8/8/8/4q3/2k4N/5B2/N1K2B2 w - - & $\mathrm{A} 3$ & -7 & +41 & -72 & $\mathrm{Z}$ & $\mathrm{u}$ \\
\hline S01 & KRKN & 8/8/8/2k1K3/8/3R4/4n3/8 w - - & {$[9$} & 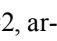 & Pazi & ;) & & \\
\hline S05 & KQKRP & 1rk5/8/8/3Q4/8/1p6/1K6/8 w - - & [9] \#( & & & & & \\
\hline $\mathrm{S} 03$ & KRKBN & $\mathrm{k} 1 \mathrm{~K} 5 / 2 \mathrm{n} 5 / 8 / 8 / \mathrm{b} 7 / 1 \mathrm{R} 6 / 8 / 8 \mathrm{w}--$ & [4] \# & 457 & 9] \#. & 5 , Nunr & & \\
\hline S05 & KRKBN & $\mathrm{k} 3 \mathrm{~b} 3 / \mathrm{n} 1 \mathrm{~K} 5 / \mathrm{R} 7 / 8 / 8 / 8 / 8 / 8 \mathrm{w}--$ & {$[9] \#$} & & & & & \\
\hline S06 & KNNKP & 8/8/1p6/1K6/2N5/3N4/8/k7 w - - & [9] \#C & & & & & \\
\hline S07 & KNPKP & 8/8/8/6Pk/4K3/4N2p/8/8 w - - & {$[9] \#^{-}$} & & & & & \\
\hline S08 & KNPKN & 8/8/8/5KPk/8/8/8/5N1n b - - & {$[9] \#^{-}$} & $7 \mathrm{a}: \mathrm{A}$ & $2 \mathrm{zug}$ & & & \\
\hline S09 & KNPKP & 8/8/8/8/4k3/7p/P3K1N1/8 w - - & [9] \# & & & & & \\
\hline $\mathrm{S} 10$ & KNPKN & 8/8/8/8/4k3/8/P3K3/5N1n w - - & [9] \# & & & & & \\
\hline S11 & KNPKPP & 8/8/8/8/5p2/4k1p1/4N1P1/5K2 w - - & {$[39] \neq$} & & Man & adler & & \\
\hline $\mathrm{S} 12$ & КРPКPP & 8/5pk1/8/2p1PK2/2P5/8/8/8 w - - & [9] \#4 & & & & & \\
\hline
\end{tabular}

Positions S01-S12 are from studies where White wins in an essentially unique way. They all feature a level A zug in both a try and the mainline solution and are mainly taken from Beasley [9]. The appendix can accommodate only a few of the solutions so there are plenty of exercises here for the reader.

${ }^{20} \mathrm{C} \equiv$ zug-significant castling rights given to $1^{\text {st }}$ or $2^{\text {nd }}$ player, $\mathrm{B} / \mathrm{C} \equiv$ Level $\mathrm{B} / \mathrm{C}$ zug, $e \equiv$ e.p. significant, and $5 \equiv 5-1 \mathrm{p}$ zug. 


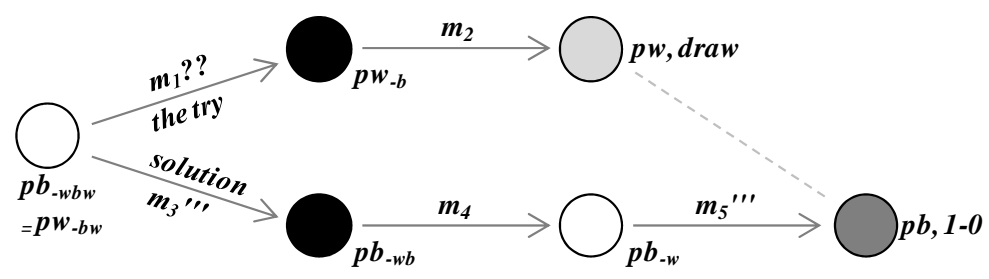

Fig. 2. The Zugzwang Study scenario.

\section{Commentary: Statistics, Gems and Studies}

Our EGT search has identified a corpus of over 900,000 zugs which may be reviewed in statistical terms, datamined for gems of various sorts, and put in the context of the Chess Study, the chess-engine and the game itself.

In addition to the Level A zug defined above, two further levels of zug are notable, q.v. Table 5. At Level B, a B1 (B2) zug is merely inconvenient, requiring the winner (loser) to make more (less) moves in some metric before some defined goal. Positions P04 (B2), N09 (B1) and S01-S12 feature examples. Note that the number of moves to goal may not be affected in all metrics. ${ }^{21}$ At Level C, zugs do not impact value or depth in any metric but the side to move would rather play a null move: Regan [27] identified P05 and Elkies [26] identified N10, both draws in which a null move eases the task of the defender. The likelihood of a win in a theoretically drawn position can be modelled using the concept of a Reference Fallible Player [28-32].

\subsection{The Statistics}

Pawnless zugs are just 5.26\% of the total; pawnful zugs account for the vast majority. There are more pawnful than pawnless positions but the presence of at least one pawn increases the density of zugs by a factor of four. Zugs are also more frequent when Knights, parity-bound and unable to lose the move ${ }^{22}$, are present.

Type A3 zugs are clearly rarer than A1-2 zugs and have been the focus to date: it had been established that no pawnless A3 zug exists with 5 men or less [11].

The presence of an en passant feature in a zug has not attracted attention so far, perhaps for three reasons. Type A1-3 zugs in EPZ remain zugs of the same type if the en passant opportunity is removed. Secondly, e.p. constrains two pawns to 14 of the 2,256 positions available and, at $0.22 \%$ of the total as in Table 3, e.p. zugs are few indeed. Finally, the very few type A4-6 zugs had not been discovered.

The next challenges are to trawl for zugs in small EGTs for positions with castling rights, and in large 7-man EGTs. Under the $D T Z_{50}$ metric which recognizes the 50move Rule, some zugs, q.v. Table 4, lose their status or change type.

${ }^{21} \mathrm{KNNKP}$ position $\mathrm{P} 04: d t z=d t c=4 \mathrm{~m}$ if a null move is available; $d t m=4 \mathrm{~m}$ regardless.

${ }^{22}$ Alternating as they do between white and black squares. 


\subsection{Datamining for Gems}

The deepest zugs come closest to not being zugs at all: the shallowest zugs feature the greatest change of advantage achievable by the null move. Relative depths may vary with the depth-metric chosen.

Rare gems have intrinsic value; zugs may be absolutely or type-unique within their endgame and/or have some rare feature. There are only 51 type A2 e.p. zugs in which position $p 3$ is shallower than position p1: 20 in KPPKP and 31 in KPPKPP.

The absence of a pawnless 5-man A3 zug naturally led to a search for one in 6-man chess. In response to the so-called Pawnless Trébuchet Challenge [33], Elkies had conjectured that position Z10, identified three years earlier [8], might just be one such: he did not claim [8] that it was as incorrectly announced by Roycroft [34]. The search turned to 6-man endgames [12], [33], [35] but the authors confirmed earlier in the work reported here that there were none [29]. Evidence of Elkies' remarkable prescience [36] is that Z10 perhaps comes closest of all 6-man pawnless positions to being an A3 zug. This is the only position found in which the $2^{\text {nd }}$ player has to avoid the loss by first playing four unique draw-saving moves. ${ }^{23}$

With KRBNKNN Z11 derived from KRNKNN Z10 [8], the A3 challenge became one of reducing the number of Knights in such a zug. The zugs B06 [29], [37], B03 [16] and B02 [38] feature two, one and no Knights respectively though B02 leaves a residual challenge by requiring obtrusive, i.e. obviously-promoted, force.

Surprisingly, there are no A3 e.p. zugs in 6-man chess but Elkies recalled an 8-man example (N01) derived from an actual game [39]. Other than the 393 A4 KPPKPP zugs, there are just three A4-6 zugs: one A4 (Z05), no A5 zugs ${ }^{24}$ and two A6 zugs (Z08-9). The A4 zug is unique in that the value of position $p 3$ is worse than the value of position $p 1$, but the $1^{\text {st }}$ player is a net winner in A5-6 zugs: the $2^{\text {nd }}$ player's perspective is irrelevant in A6 zugs. Elkies has provided the first known A5 zug (Z07) and examples N02-3 of an added e.p. opportunity dezugging a zug.

Castling rights have not been included in EGTs. However P01 and P02 are the first known zugs where added castling rights dezug or not. Elkies [26] provided exemplar zugs (N04-8) where the provision of $0-0(-0)$ castling rights to the $1^{\text {st }}$ or $2^{\text {nd }}$ player creates a zug: some also feature a significant e.p. opportunity.

\subsection{Zugzwangs and Studies}

In the Chess Study, White is by convention challenged to draw or win. The appearance of a zugzwang position in a study is notable in itself and, if it is Black to move, suggests that White is just one ply from missing its objective. Mandler's study S11 [40] requires White to revisit a previous physical position 11 plies later but with Black to move: that position is therefore a Level B zug.

A Zugzwang Study is defined to be one in which the zugzwang not only appears in the main line of a study in White's favour but also appears as the refutation of a plausible sideline try [9]. Fig.2 illustrates the requirements for such a study: a position $p$

${ }^{23} 1$.... Nc5' 2. Nd4 Ne3+' 3. Kd2 Kf2' 4. Ne6 \{other moves pressure more\} Nxe6'.

${ }^{24}$ And MB [16] reports that KPPPKPP* (assuming only $\mathrm{P}=\mathrm{Q}$ allowed) has no A5 zugs. 
must appear in its wtm form $p w$ in the try and in its btm form $p b$ in the main line; White's moves should be essentially unique and Black should play its 'best defence'. Beasley gives some remarkable examples of the genre and his article on the theme, from which most of the study positions S01-S12 are taken, is recommended. The zugzwang study demonstrates that the aesthetic contribution [41] of a zugzwang position to a study must be judged in the context of that study and not in isolation.

\section{Summary}

The authors have searched the available Nalimov DTM EGTs for 6-man chess to identify all the Level A zugzwangs. Somewhat accidentally, we have discovered three new types of zugzwang to make six types in all: there are no other types.

Work will turn to zugs in the more recently arrived 6-man 'DTC' results [16] which will be compared with those of Nalimov ${ }^{25}$ and Thompson [12], to Level B and C zugs, and to an examination of the occurrence of zugs in studies [42-43].

Complementing this review, the full results, including statistics, highlights and lists of all the zugs with their DTM depths, are published on the ICGA website [17]. The zugs may be studied using EGT query services on the web [13], [44] and we look forward to them being mined for gems by the Chess Studies community and others.

Acknowledgments. Our thanks first to Eugene Nalimov for his major contribution of 3- to 6-man DTM EGTs, to Andrew Kadatch for the software used to compress them, and to Marc Bourzutschky, Nelson Hernandez and Kyrill Kryukov for seeding their promulgation. Secondly, our thanks to Marc for his GTBGEN generalization of Nalimov's TBGEN used to identify the zugs, for his 5-1p and 7-man zugs and for his continued interest. Thanks go to Noam Elkies for a remarkable string of 'first exemplar' zugs involving e.p., castling or 5-1p chess. Finally, thanks to Ken Regan and Noam for the first known Level C zugs, and to John Beasley, Harold van der Heijden, Philip Henderson, Soren Riis and John Tamplin for stimulating dialogue.

\section{References}

1. Nalimov, E., Haworth, G.M ${ }^{\mathrm{c}}$ C., Heinz, E.A.: Space-efficient Indexing of Endgame Tables for Chess. ICGA J. 23-3, 148--162 (2000)

2. Kryukov, K.: EGTs Online. http://kirill-kryukov.com/chess/tablebases-online/ (2006)

3. Roycroft, A.J.: Test Tube Chess: A Comprehensive Introduction to the Chess Endgame Study. Faber and Faber Ltd. (1972)

4. Nunn, J.: Secrets of Pawnless Endings. B.T. Batsford, London. $2^{\text {nd }}$ edition (2004)

5. Nunn, J.: Secrets of Rook Endings. B.T. Batsford, London. $2^{\text {nd }}$ edition. Gambit (1999)

6. Nunn, J.: Secrets of Minor-Piece Endings. B.T. Batsford, London (1995)

7. Beasley, J., Whitworth, T.: Endgame Magic. B.T. Batsford, London (1996)

8. Elkies, N.D.: No. 10965: mutual full-point zugzwang? EG 8-128, 320 (1998)

9. Beasley J.: Creating reciprocal zugzwang studies. EBUR 12-2, 8--12 (2000)

${ }^{25}$ First indications [31] are that the 'DTC' and 'DTM' statistics are in full agreement. 
10. Wirth, C., Nievergelt, J.: Exhaustive and Heuristic Retrograde Analysis of the KPPKP Endgame. ICCA J. 22-2, 67--80 (1999)

11. Haworth, G.M ${ }^{\mathrm{C}}$ C., Karrer, P., Tamplin, J.A., Wirth, C.: 3-5-Man Chess: Maximals and Mzugs. ICGA J. 24-4, 225--230 (2001)

12. Tamplin, J., Haworth, G.M ${ }^{\mathrm{c}}$ C.: Ken Thompson's 6-man Tables. ICGA J. 24-2, 83--85 (2001)

13. Bleicher, E.: Endgame Service based on Nalimov's EGTs http://www.k4it.de/index.php?topic $=$ egtb\&lang $=$ en $(2009)$

14. Tay, A.: A Guide to Endgame Tablebases. http://www.horizonchess.com/FAQ/Winboard/egtb.html (2009)

15. Konoval, Y., Bourzutschky, M.S.: Private Communications (2007-8)

16. Bourzutschky, M.S.: Private Communications (2009)

17. ICGA: The ICGA website. Menu: Game-specific information - Western Chess - Endgames. www.icga.org (2009)

18. Thyen, O., Clark, M., Scholze-Stubenrecht, W., Sykes, J.B.: The Oxford-Duden German Dictionary. OUP (2005)

19. Hooper, D., Whyld, K.: The Oxford Companion to Chess. OUP (1992)

20. Gijssen, G.: An Arbiter's Notebook: Monroi and Other Matters. Chesscafe.com http://www.chesscafe.com/text/geurt105.pdf (2006)

21. Gijssen, G.: An Arbiter's Notebook: Interpreting the Rules. Chesscafe.com http://www.chesscafe.com/text/geurt110.pdf (2007)

22. Gijssen, G.: An Arbiter's Notebook: Interpreting the Rules. Chesscafe.com http://www.chesscafe.com/text/geurt129.pdf (2009)

23. Bourzutschky, M.S.: The 16 "missing” Nalimov files. http://preview.tinyurl.com/aqevp2 (2006-07-18)

24. Hernandez, N.: 'Missing 16 received!'. Private Communication (2006-08-08)

25. Bourzutschky, M.S.: Tablebase comparison. http://www.tinyurl.com/d3wny4 (2006-08-10)

26. Elkies, N.D.: Private Communications (2009)

27. Regan, K.W.: Private Communications (2009-04)

28. Haworth, G.M ${ }^{\mathrm{c}}$ C.: Reference Fallible Endgame Play. ICGA J. 26-2, 81--91 (2003)

29. Haworth, G.M ${ }^{\mathrm{c}}$ C.: Chess Endgame News. ICGA J. 28-4, 243 (2005)

30. Haworth, G.M ${ }^{\mathrm{c}}$ C.: Gentlemen, Stop Your Engines! ICGA J. 30-3, 150--156 (2007)

31. Di Fatta, G., Haworth, G.M ${ }^{\mathrm{c} C}$., Regan, K.W.: Skill Rating by Bayesian Inference. In: Proc. IEEE Conf. on Computational Intelligence and Data Mining, Nashville, USA, 89--94 (2009)

32. Haworth, G.M ${ }^{\mathrm{c}}$ C., Di Fatta, G., Regan, K.W.: Performance and Prediction: Bayesian Modelling of Fallible Choice in Chess. In: Proc. Advances in Computer Games 12. Springer LNCS (2009)

33. Roycroft, A.J.: Announcement of the Pawnless Trébuchet task. EG 7-116, 633 (1995)

34. Roycroft, A.J.: Report 1 on the Pawnless Trebuchet task. EG 7-117, 645 (1995)

35. Costeff, G.: EG 1-152 online. http://www.gadycosteff.com/eg/eg.html (2009)

36. Beasley, J.: Gems Discovered by Computer. BESN Special Number 46, 6--7 (2005)

37. Bourzutschky, M.S., Konoval, Y.: 7-man Endgame Databases. EG 11-162, 493--510 (2006)

38. Haworth, G.M ${ }^{\mathrm{c} C}$.: Chess Endgame News. ICGA J., 29-2, 79 (2006)

39. Elkies, N.D.: On Numbers and Endgames: Combinatorial Game Theory in Chess Endgames. In: Games of No Chance (ed. R.J. Nowakowski), MSRI 29 (1996)

40. Beasley, J.: Depth and Beauty: The chess endgame studies of Artur Mandler (2003)

41. Iqbal, A.: A Discrete Computational Aesthetics Model for a Zero-Sum Perfect Information Game. http://metalab.uniten.edu.my/ azlan/Misc/phd_thesis_azlan_(final).pdf Ph.D. thesis. University of Malaya, Kuala Lumpur, Malaysia (2008)

42. Costeff, G., Stiller, L.B.: Chess Query Language CQL. http://rbnn.com/cql/ (2003)

43. Van der Heijden, H.: Endgame Study Database III, 67,691 Studies (2005)

44. Tamplin, J.A.: Multimetric endgame service. http://chess.jaet.org/endings/ (2009) 


\section{Appendix: Some Zugzwang Lines}

The 7-man lines are from Bourzutschky [16], [37]. All moves are at least optimal moves given the move-selecting strategy nominated, and beyond that, the key is:

"'" $\equiv$ only value-saving move (independent of move-choosing strategy),

$" \equiv$ the only optimal move, given the strategy nominated, e.g., $\mathrm{SC}^{-} \mathrm{M}^{-}$, and

${ }^{\circ} \equiv$ only move.

KPPKP Z04: positions $p 1$ and $p 3$ have different depths to mate.

p1, btm: $\{d t m=25\} \mathrm{SM}^{-} / \mathrm{SM}^{+} 1$.... cxd3" 2. Kxd3"'" Kc5" 3. Kc3"'" Kb5" 4. Kb3"'"

Ka5 5. Kc4" Kb6" 6. Kb4""' Kc6 7. Ka5" Kb7" 8. Kb5" Ka7" 9. Kc6" Ka8" 10. b4 $\mathrm{Kb}^{\prime \prime}$ 11. Kb6" Ka8" 12. b5 Kb8 ${ }^{\circ}$ 13. Ka6" Kc7" 14. b6 Kc8" 15. Ka7"'" 1-0

p2, wtm: $1 . \mathrm{K} \sim \mathrm{Kxd} 4=$ or $1 . \mathrm{b} \sim \mathrm{cxb}^{\prime \prime \prime \prime}=$

p3, btm: $\{d t m=15\} \mathrm{SM}^{-} / \mathrm{SM}^{+} 1 \ldots \mathrm{K}(\mathrm{c} 6 / \mathrm{d} 6 / \mathrm{e} 6) 2 . \mathrm{Kxc}^{\prime \prime}\{d t c=7\} 1-0$

KBPKPP Z05: an A4, draw-win-loss, zug:

p1, btm: 1. ... cxb3"'"' 2. Bxb3"'" =

p2, wtm: $\mathrm{SM}^{+} \mathrm{Z}^{+} / \mathrm{SM}^{-} \mathrm{Z}^{-} 1 . \mathrm{Ke}^{\prime \prime} \mathrm{c}^{\prime \prime} 2 . \mathrm{Bb} 3 \mathrm{c} 2^{\prime \prime} 3 . \mathrm{K} \sim \mathrm{K}(\mathrm{b} 1 / \mathrm{d} 1 / \mathrm{d} 2)$ 4. Bxc2" $\mathrm{Kxc} 2^{\prime \prime \prime \prime}$ 5. Ke1 K(b3/c3) 6. Kd1 Kxb4"'" 0-1

p3, btm: $\mathrm{SMZZ}^{-} / \mathrm{SM}^{+} \mathrm{Z}^{+} 1$.... Kb1" 2. Ke3" Kc1" 3. Be2 Kc2" 4. Kd4"'" c3" 5. Bd3+" Kd2" 6. Be4 c2" 7. Bxc2"'” Kxc2" 8. Kc5"'" Kb3 9. Kxb5"'” Ka3 10. Kc5 Kb3 11. b5" 1-0

KRBNKNN Z11: an A3 zug adaption of Z10 which 'just failed' to be A3.

$p 1 \equiv p 3$, wtm: $1 . \mathrm{N} \sim \mathrm{N}(\mathrm{x}) \mathrm{d} 3 \#^{\prime \prime \prime \prime} ; 1$. R Nxb2\#"'" 0-1

p2, btm: ‘"Black cannot maintain the bind" [8]\} 1... Nc5 2. Nd4 Ne3+3. Kc1 Kg1 4. Bf5 Ng2 5. Nf3+ Kf1' 6. Bh3 Nd3+' 7. Kb1 Nb2 8.Kxb2 $\{d t c=1 \mathrm{~m}, d t m=8 \mathrm{~m}\}$

KNNNKNN B01: the bK is boxed in but White must avoid a KNNK endgame. p2, btm: $\{d t z=17\} \mathrm{SZ}^{-} / \mathrm{SZ}^{+} 1 \ldots \mathrm{Ndc} 3$ 2. Nd7"'" Kh7 3. Nf4"'" Kg8 4. Nd6"'" Kg7 5.

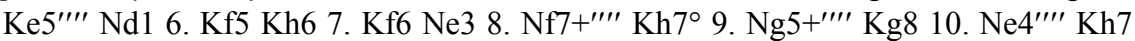
11. Ne5 Nb6 12. Kf7"'" Nbc4 13. Nf6+"'"' Kh6 14. Nf3"'" Nd6+ 15. Kf8 Nef5 16. $\mathrm{Ng} 8+\mathrm{Kh}^{\circ}$ 17. $\mathrm{Ng} 5+\mathrm{Kh}^{\circ} 18 . \mathrm{Ng} 6 \#\{10$ of White's 17 moves were unique winning moves $\} 1-0$

KRBBKQN B03: an A3 zug with only one Knight.

p1, wtm: SZ ${ }^{+} / \mathrm{SZ}$ 1. Be8 Ka2 2. Bf7 Ka3 3. Bc1+ Kb4 4. Bd2+ Kc4 5. Be8 6.Ne7 0-1

$\{1$. Bxd5 Qxg6+"'" $\{\mathrm{KBBKQ}, d t z=62)$ "is however probably best defence" [16]\}.

p2, btm: $\mathrm{SM}^{-} Z^{-} / \mathrm{SM}^{+} Z^{+} 1$. ... Qh3 2. Ra6+" Qa3 ${ }^{\circ}$ 3. Rxa3\#" 1-0

KRKN S01: 1.Re3"'" Ng1' 2.Kf5' (2.Kf4?? Kd4 pw =) Kd4' 3.Kf4"'" pb 1-0

KRKBN S03: 1.Rb6"'" Nb5' 2.Ra6+"'" Na7+ 3.Kc7"'" Be8' 4.Ra3"' (4.Ra2?? Ba4"'" pw $=) \mathrm{Ba}^{\prime}$ ' 5.Ra2' pb 1-0

KPPKPP S12: 1.Kf4"'" (1.Ke4?? Kg6" pw) Kg6' 2.Ke4"'" pb Kg5' 3.e6"'" fxe6' 4.Ke5"'" Kg4 5.Kxe6"'" 1-0 